\title{
Estradiol Cypionate
}

National Cancer Institute

\section{Source}

National Cancer Institute. Estradiol Cypionate. NCI Thesaurus. Code C47991.

The cypionate salt form of estradiol, the most potent, naturally produced estrogen.

Estradiol cypionate diffuses through the cell membrane and binds to and subsequently activates the nuclear estrogen receptor found in the reproductive tract, breast, pituitary, hypothalamus, liver, and bone. The activated complex binds to the estrogen response element on the DNA and activates the transcription of genes involved in the functioning of the female reproductive system and secondary sex characteristics. 